\title{
A Novel Approach for Fault Tolerance in Ad-Hoc on Demand Protocol
}

\author{
Abhilash Sharma \\ BBSBEC, Fatehgarh Sahib, India
}

\begin{abstract}
MANETs are multi-hop ad-hoc wireless networks where nodes can move arbitrary in the topology with variation of speed and trajectory. As MANET network has no infrastructure or dependency, it can be implemented easily in any environment. But the main problem in such networks is to calculate the behavior of different routing protocols in different environments due to limited computing power, low bandwidth, high mobility and absence of central coordinating entity. The need for quality of service in mobile Adhoc infrastructure is increasing day by day due to more attacks, faults and congestion. Two major parts which provide such kind of service in mobile Adhoc devices are fault tolerance and energy saving. This proposed work is suitable for fault tolerance of the node in MANET by applying a novel cluster approach and time constraints. This work also aimed at implementing battery checking scheme to avoid faulty conditions in network and to provide an auxiliary path finding scheme in case of node failure. The simulated results illustrate that the proposed algorithm performs well as compared to existing techniques for various parameters.
\end{abstract}

\section{Keywords}

MANET, AODV protocol, Clustering Approach, Fault Tolerance, TAODV

\section{INTRODUCTION}

MANETs are the networks which comprises of mobile nodes, a router with multiple hosts and wireless communication devices. Such networks consist of wireless mobile nodes which can freely and dynamically self organized into arbitrary and temporary Adhoc network topologies. The wireless communication devices are transmitters, receivers and smart antennas; where antennas can be of any kind. Nodes may be a mobile phone, laptop, personal digital assistance, MP3 player and personal computer and it can be fixed or mobile.

MANET is a spontaneous network. It is helpful when dealing with wireless devices in which some of the devices are part of the network only for the duration of a communication session. The major characteristics and challenges of MANETs are as follows:

\section{Cooperation:}

This is one of the important characteristic of MANETs as it is helpful for the communication of source node and destination node when they are out of range with each other. In such scenario, the communication between both the nodes takes place with the cooperation of other nodes.

\section{Dynamism of Topology:}

The nodes of MANET are randomly, frequently and unpredictably mobile within a network. As these nodes may leave or join the network dynamically; it

\author{
Birinder Singh \\ BBSBEC, Fatehgarh Sahib, India
}

significantly affects the status of trust among nodes and complexity of routing. Thus, the topology of the network is unpredictable.

\section{Lack of fixed infrastructure:}

Since there is no fixed or centralized infrastructure in MANETs, so there is no need to establish a centralized authority to control the network characteristics.

This work is an attempt to provide a novel approach for fault tolerance in MANETs and to increase its efficiency in comparison to existing techniques.

\section{RELATED WORK}

Adeel Akram et al in [2] explained that battery capacity has become a critical factor for continuous ubiquitous connectivity. Wireless mesh nodes operate with finite battery capacity which is to be managed efficiently to increase reliability of individual nodes and overall lifetime of the network. This paper introduces a modified AODV routing protocol for battery and frequency optimization to be employed in Wireless Mesh Networks (WMN). Further the isolation of nodes with critically low battery power, periodic announcements of battery capacities, and occasional rechecking of critical nodes' reliability increases the overall lifetime of unreliable WMN. In WMN, the key challenge is the efficient utilization of network resources of nodes in particular and for the network in general. The approach is to isolate critical nodes before they further deteriorate the network throughput. Energy optimized AODV is implemented from route discovery to route maintenance to the discovery of critical nodes.

N.Jaisankar et al in [12] explained that Due to limited transmission range of wireless network nodes, multiple hops are usually needed for a node to exchange information with any other node in the network. Thus routing is a crucial issue in the design of MANET. Nodes will use a flood-based discovery mechanism to find routes when required. The wide availability of wireless devices requires the routing protocol should be scalable. But, as the size of the network increases the on demand routing protocols produce poor performance due to large routing overhead generated while repairing route breaks. In this paper authors discussed about the multiple paths in a single path. Multipath routing can provide load balancing and reduce the frequency of on-demand route discovery.

Mamatha et al [19] explains about the several MANET constraints such as limited bandwidth, mobility, battery power etc; it becomes very important to design a protocol that suits the requirements for MANETS. In this paper, authors suggest a protocol mechanism which is loosely based on a reactive protocol AODV (Ad Hoc On demand Distance Vector Protocol). Authors proposed protocol uses the time concept based on first come first served basis for path choosing 
process, hence the name Time On Demand Distance Vector Protocol (TODV). The protocol design presented here suits the MANETS dynamic topology perfectly in finding the best path or route for data communication. The simulation study reveals that the proposed protocol outperforms than existing AODV, in terms of throughput and end-to-end delay.

Kaur et al [13] explained that Wireless local area networks (WLANs) are in a period of great expansion and there is a strong need for them to support multimedia applications. With the increasing demand and penetration of wireless services, users of wireless networks now expect Quality of Service (QoS) and performance comparable to what is available from fixed networks. Providing QoS requirements like good throughput and minimum access delay are challenging tasks with regard to 802.11 WLAN protocols and Medium Access Control (MAC) functions. Authors particularly explained about the quality of service and explained that QoS is directly proportional to the fault occurrence in the network so highlight the importance of fault tolerance in MANETs.

Che-Aron et al [6] elaborates that wireless sensor networks suffer from resource limitations, high failure rates and faults caused by the defective nature of wireless communication and the wireless sensor characteristics. In this paper, they address the reliability issue by designing and developing an enhanced fault-tolerance mechanism of Ad hoc On-Demand Distance Vector (AODV) routing protocol for WSN called the Enhanced Fault-Tolerant AODV (ENFAT-AODV)routing protocol. The proposed ENFAT-AODV routing protocol improves the reliability and robustness of the network by creating a backup path for every node on a main path of data delivery. When the node gets failure to transmit a data packet through the main path, it immediately utilizes its backup route to become a new main path for the transmission of next coming data packets without any interruption.

\section{PROPOSED WORK}

The quality of service is the critical factor in MANETs. Fault tolerance and energy saving are most essential parts for providing quality of service in Mobile ad-hoc devices. Moreover fault tolerance based on time constraints is needed to be explored which demand refined and stable fault tolerance mechanism. The energy saving mechanism is done by improving quality of service by avoiding unwanted usage of energy and node failures in between wireless nodes by introducing sleep mode after specific interval of time. This research reflects the link failure and provides solutions for energy saving by switching to energy saving mode that is sleep mode (when node is not in use). Moreover, it is based on the Adhoc Time based on demand distance vector protocol so that the energy saving can be done. It particularly works on the detection of faulty nodes at the early state of the node connectivity. It finds out the nodes which have low resources to sustain in whole process of routing and by removing these nodes, we can filter out the weak links from whole topology which in turn, can enhance the quality and fault tolerance. At initial state after finding faulty nodes (Nodes with very less battery resources), these nodes will be in sleep mode and will not participate in routing process. After shredding those nodes we will proceed with other mechanisms based on time constraints. Quality of the network will be the final target as quality is directly link to the amount of faults. Parameters for fault tolerance are throughput, packets received, packets drop and delay.

\section{SIMULATION MODEL}

To simulate the results Opnet 14.5 is used. This research has focused on to present better solution for power saving by improving quality in selection of nodes which are best fitted for routing in between wireless nodes. This research reflects the link failure and provides solutions for it. This work focused on the Adhoc Time based on demand distance vector protocol so that the faulty networks can be prevented. Initially work started with battery resources as the prime factor in deciding the proposed work and later the search for other dependent factors like refined routing table, topology management etc. At earlier stage the nodes having less battery resources are find out from the network and will not allow participating in the network. After removing those nodes from network the work proceed with other mechanism based on time constraints and routing techniques.

Research has started with building a MANET network in Opnet simulator with FTP traffic for measurement of results. In the proposed work, three scenarios had been designed i.e. simple AODV, sleep mode and clustered approach. For battery checking and sleep mode setup, time to live has been modified and Receiver module has been used in simulation. Basically in this work restrict the gap in TTL and Receiver module is used to carry information of previous nodes for route finding which is very helpful in finding new route.

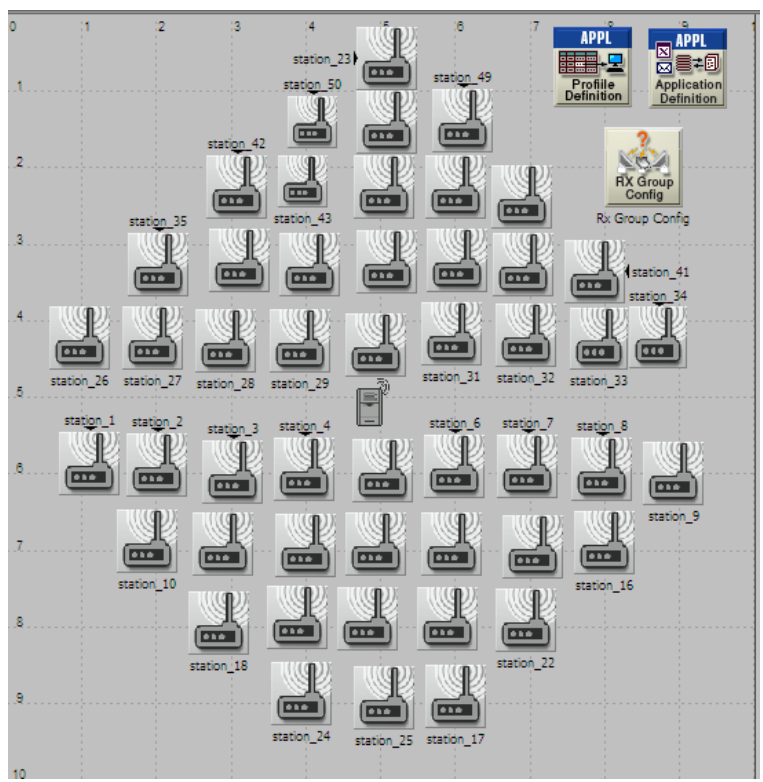

Fig 1: Network Topology used for sleep mode

Further cluster head nodes have been selected for making cluster environment. Cluster head are two in number and fetch data from other nodes. In network topology, nodes close to server act as cluster heads. 


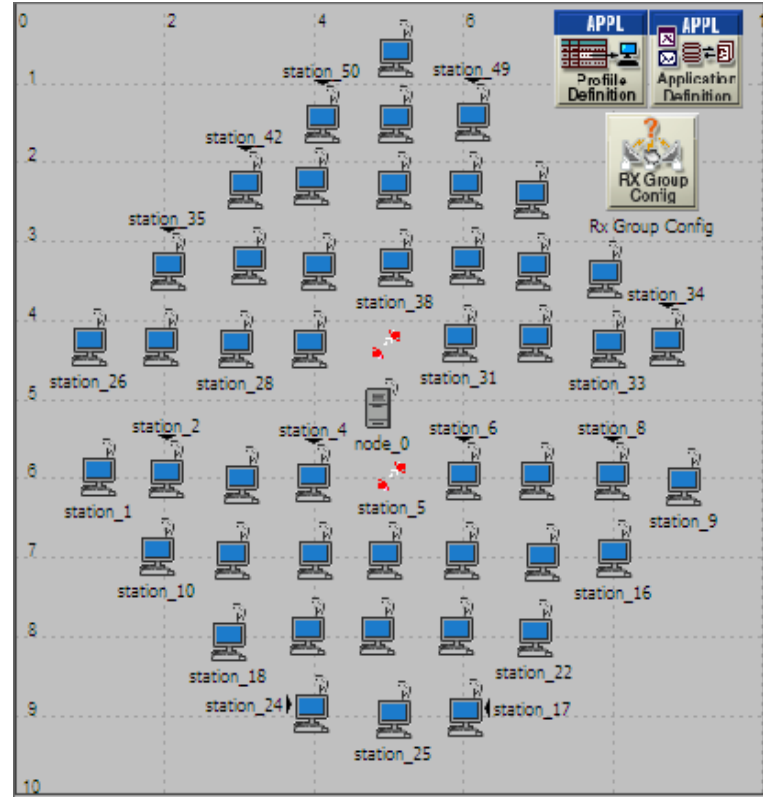

Fig 2: Network Topology for Clustered scenario

Cluster heads have special configuration and have better data rate and RTS threshold values as compared to the other devices.

\begin{tabular}{|c|c|c|c|c|}
\hline \multicolumn{4}{|c|}{ * (station_30) Attributes } & $\square$ 回 $x$ \\
\hline \multicolumn{5}{|c|}{ Type: workstation } \\
\hline \multicolumn{2}{|c|}{ Attribute } & \multirow{2}{*}{\multicolumn{2}{|c|}{$\begin{array}{l}\text { Value } \\
\text { Auto Assigned }\end{array}$}} & \multirow[t]{2}{*}{$\Delta$} \\
\hline (3) & Wireless LAN MAC Address & & & \\
\hline (2) [ & - Wireless LAN Parameters & $(\ldots)$ & & \\
\hline (3) & BSS Identfier & \multicolumn{2}{|l|}{ Auto Assigned } & \\
\hline (2) & - Access Point Functionality & \multicolumn{2}{|l|}{ Disabled } & \\
\hline (2) & Physical Characteristics & \multicolumn{2}{|c|}{ Direct Sequence } & \\
\hline (2) & Data Rate (bps) & \multicolumn{2}{|c|}{$11 \mathrm{Mbps}$} & \\
\hline (2) & $\boxplus$ Channel Settings & \multicolumn{2}{|l|}{ (...) } & \\
\hline (3) & Transmit Power $(W)$ & \multicolumn{2}{|l|}{0.001} & \\
\hline (?) & - Packet Reception-Power Threshold... & \multicolumn{2}{|l|}{.90} & \\
\hline (?) & Rits Threshold (bytes) & \multicolumn{2}{|l|}{256} & \\
\hline (2) & Fragmentation Threshold (bytes) & \multicolumn{2}{|l|}{ None } & \\
\hline (2) & CTS to-self Option & \multicolumn{2}{|l|}{ Enabled } & \\
\hline (3) & . Short Retry Limit & \multicolumn{2}{|l|}{7} & \\
\hline (2) & Long Retry Limit & \multicolumn{2}{|l|}{4} & \\
\hline (3) & AP Beacon Interval (secs) & \multicolumn{2}{|l|}{0.02} & \\
\hline (3) & Max Receive Lifetime (secs) & \multicolumn{2}{|l|}{0.5} & \\
\hline (3) & Buffer Size (bits) & \multicolumn{2}{|l|}{256000} & \\
\hline (3) & Roaming Capability & \multicolumn{2}{|l|}{ Disabled } & \\
\hline (2) & Large Packet Processing & \multicolumn{2}{|l|}{ Drop } & \\
\hline (?) & Ф PCF Parameters & \multicolumn{2}{|l|}{ Disabled } & \\
\hline (3) & $\oplus$ HCF Parameters & \multicolumn{2}{|l|}{ Not Supported } & $\nabla$ \\
\hline \multicolumn{2}{|c|}{ (3) } & \multirow[t]{2}{*}{ Filter } & \multicolumn{2}{|c|}{$\begin{array}{r}\Gamma \text { Advanced } \\
\Gamma \text { Apply to selected objects }\end{array}$} \\
\hline \multicolumn{2}{|c|}{$\Gamma$ Exact match } & & $\underline{Q K}$ & Cancel \\
\hline
\end{tabular}

Fig 3: Configuration of Cluster head nodes

Research started with basic parameters which are explained below in table 1 .

\begin{tabular}{|cc|}
\hline Attributes & Values \\
\hline Channel & Wireless \\
\hline Transmission & File Transfer Protocol \\
\hline Routing Protocol & AODV \\
\hline Start time(sec) & $\mathbf{0}$ \\
\hline End Time(sec) & $\mathbf{1 0 0}$ \\
\hline File Size (bytes) & $\mathbf{5 1 2}$ \\
\hline Number of Nodes & $\mathbf{5 0}$ \\
\hline Delay(optimal) & $\mathbf{0 . 0 1}$ \\
\hline Packet send & $\mathbf{1 0 0 0 0}$ \\
\hline
\end{tabular}

Table 1: Basic parameter for simulation

\section{FAULT TOLERANCE MODEL}

In this model, power status of nodes are measured by route table according to the clustered formed for new routes. The nodes which have active status or critical status are checked and certain path is formed within specific time limit. If a node takes more time to respond back then that node is not allowed to participate in the network for route establishment. 


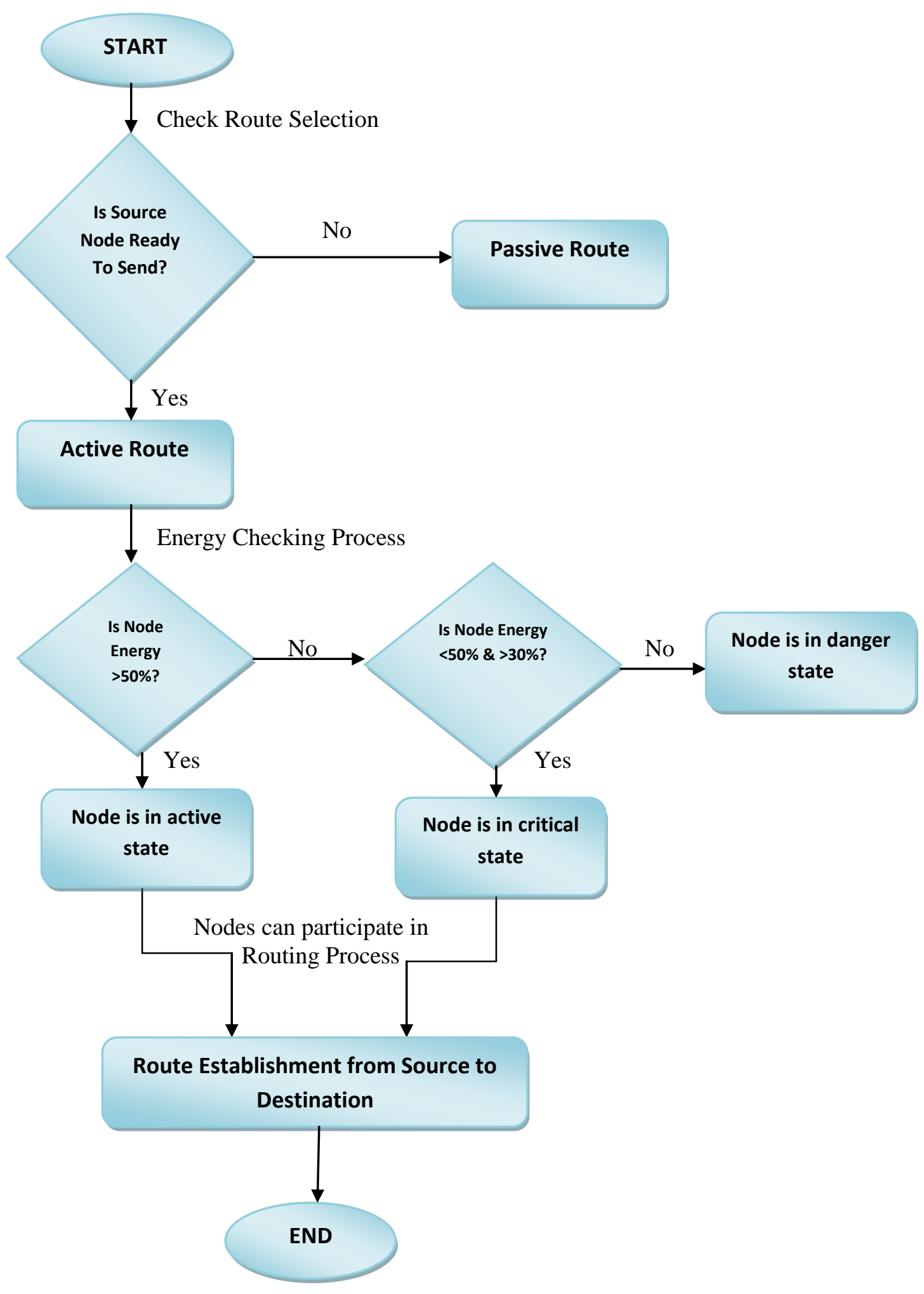

Fig 4: Fault Tolerance Model

\section{RESULT DISCUSSIONS}

In this work, Quality of service of AODV is measured with parameters like packets received, packets drop, throughput and delay under the different environments like simple AODV, sleep mode and clustered network. The network topologies used for experimentation are Normal AODV, Sleep mode scenario, proposed scheme with Clustered approach and failure of nodes network scenario. In this section, all the simulation results are discussed and results are shown in form of graph. 
Results obtained for normal performance of AODV, Performance of Sleep mode concept, Performance of Clustered approach in sleep mode concept and performance behavior of Faulty network in terms of throughput, delay, Packets received and packets drop are discussed in the following sections.

\section{a. Performance of three scenarios with Throughput}

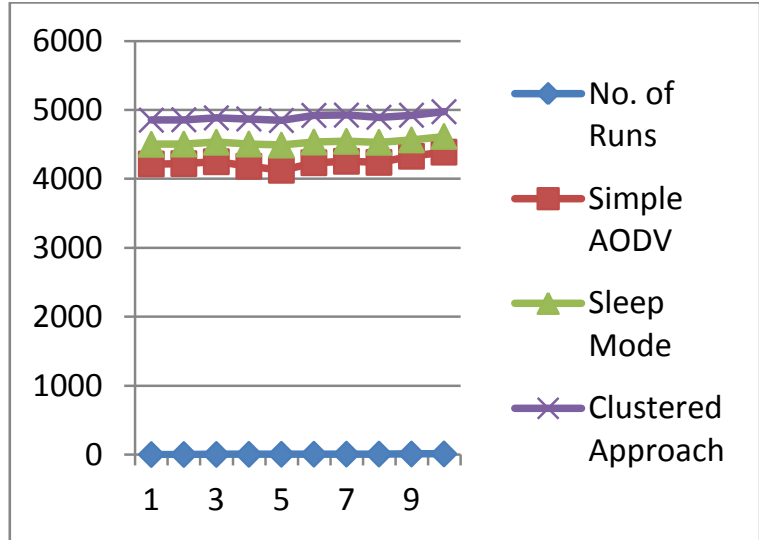

Fig 5: Throughput (bits/sec) comparisons of all three scenarios

\section{b. Performance of three scenarios with Delay}

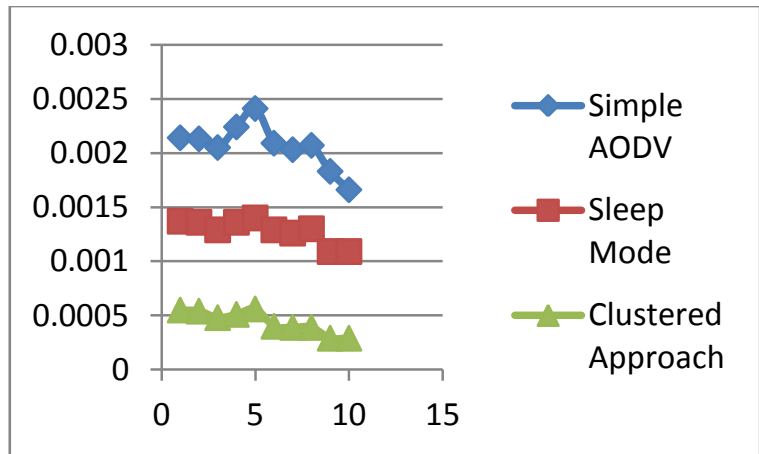

Fig 6: Delay (sec) comparisons of all three scenarios

\section{c. Performance of three scenarios with packets receive}

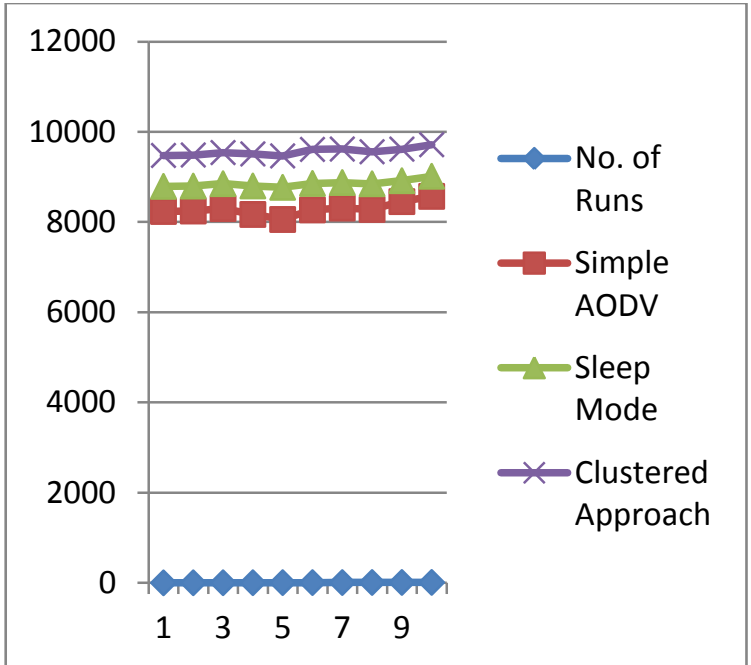

Fig 7: Packets receive comparison of all three scenarios

\section{d. Performance of three scenarios with packets drop}

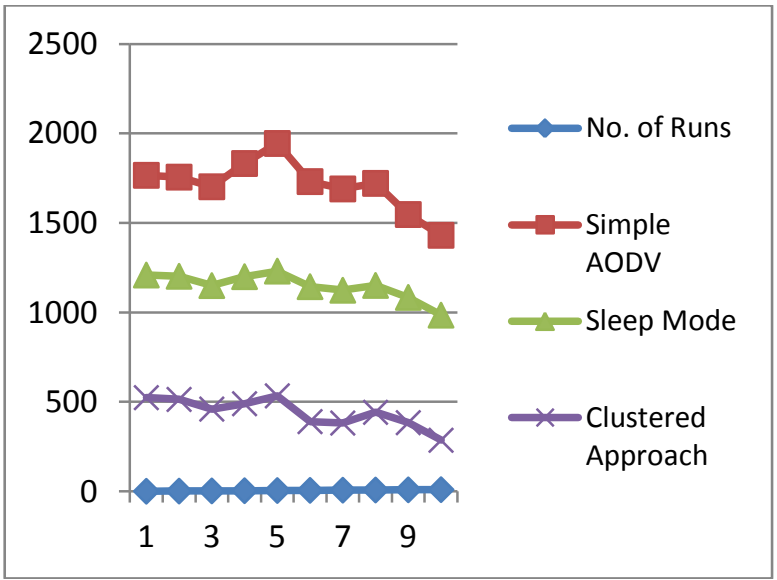

Fig 8: Packets drop comparison of all three scenarios

The overall simulation performance is presented in table 2, which shows that the clustered network provides the better results as compared to sleep mode and simple AODV scenarios where as fault tolerance value is directly proportional to the throughput value of the network as the number of faults increases, it affects the network performance. The overall simulation runs for $100 \mathrm{sec}$ for ten times then the average results are calculated which shows better result for the clustered network in all the parameters like throughput, delay, packets received, packets drop. 


\begin{tabular}{|c|c|c|c|}
\hline Attributes & $\begin{array}{c}\text { Simple } \\
\text { AODV }\end{array}$ & $\begin{array}{c}\text { Sleep } \\
\text { mode }\end{array}$ & $\begin{array}{c}\text { Clustered } \\
\text { Approach }\end{array}$ \\
\hline $\begin{array}{c}\text { Throughput } \\
\text { (bits/sec) }\end{array}$ & 4243.48 & 4532.17 & 4894.34 \\
\hline Delay (sec) & 0.0020 & 0.0012 & 0.0004 \\
\hline $\begin{array}{c}\text { Packets } \\
\text { receive }\end{array}$ & 8288 & 8852 & 9559 \\
\hline Packets drop & 1712 & 1148 & 441 \\
\hline
\end{tabular}

Table 2: Result summary

\section{CONCLUSIONS \& FUTURE SCOPE}

After the simulation results we concluded that, the performance of the Ad-hoc on demand distance vector routing protocol has been summarized. The main focus is to show the performance of AODV under three scenarios like normal environment, under Clustered environment and under sleep mode in term of throughput, delay, packets received and packets drop. In doing so, a Sleep Mode Concept scenario has been created and performance found to be better than normal AODV. This network further extended to create cluster heads and cluster formation according to the energy check level. Performance of network increases after clustering according to the proposed work.

It is an important issue for the further study to implement the proposed scheme on the distributed environment. Various types of TCP protocols such as TCP Reno, TCP Vegas and TCP FRC needs good testing for the given scheme. The proposed work need strong testing in scenario where congestion is more and priority wise distribution of traffic should need to be considered. Moreover implementation of clustering approaches with proposed scheme can be considered for providing security with resources saving in the wireless Ad-hoc networks.

\section{REFERENCES}

[1] Aitha Nagaraju and Ramachandram Srinadas," A Strategy to Reduce the Control Packet Load of AODV Using Weighted Rough Set Model for MANET", The International Arab Journal of Information Technology, pp. 77-82, Volume- 8, No. 1, January 2011.

[2] Akram Adeel, Mariam Shafqat, "Battery and Frequency Optimized AODV for Wireless Mesh Networks", Canadian Journal on Multimedia and Wireless Networks Volume- 1, No. 3, pp 569-571, April 2010.

[3] Balachandran Krishna, Wing Cheong Lau and Joseph H. Kang, " Adaptive Sleeping and Awakening Protocol (ASAP) for Energy Efficient Adhoc Sensor Networks", 0-7803-8938-7/05/\$20.00 (C) 2005 IEEE.

[4] Belalem Ghalem, Esma Insaf Djebbar, Abderahmann Benaissa, Ali Cherif Brakeche," Service for fault tolerance in the Ad Hoc Networks based on Multi Agent Systems", Computer Science Journal of Moldova, volume-18, Issue-3, pp 54-56, 2010.

[5] Cao Lijuan, Kashif Sharif, Yu Wang and Teresa Dahlberg, "Adaptive Multiple Metrics Routing Protocols for heterogeneous Multi-Hop Wireless Networks', Department of Computer Science, University of North Carolina at Charlotte, Charlotte, USA.

[6] Che-Aron Zamree, Wajdi Al-Khateeb and Farhat Anwar, "The Enhanced Fault-Tolerance Mechanism of AODV Routing Protocol for Wireless Sensor Network", IJCSNS International Journal of Computer Science and Network Security, Volume-10 No.6, June 2010.

[7] Chlamtac Imrich, "Mobile ad hoc networking: imperatives and challenges", Faculty of Computing Sciences and Engineering De Montfort University Leicester, LE1 9BH, UK.

[8] Dai Fei and Jie Wu, "Efficient Broadcasting in Ad-Hoc Networks using Directional Antennas", Department of Computer Science and Engineering, Florida Atlantic University, Boca Raton, FL 33431.

[9] Ezhilchelvan Paul D and Einar W Vollset," Design and Performance-Study of Crash Tolerant Protocols for Broadcasting and Reaching Consensus in MANETs", Proceedings of the 2005 24th IEEE Symposium on Reliable Distributed Systems (SRDS'05).

[10] Gupta Jyoti," Fault Tolerant Wireless Mesh Network: An Approach", International Journal of Computer Applications, Volume- 23, issue- 3, pp 43-46, June 2011.

[11] Hosseini Seyed Ali, Hamid Farrokhi, PhD," The Impacts of Network Size on the Performance of Routing Protocols in Mobile Ad-Hoc Networks", Second PacificAsia Conference on Circuits, Communications and System (PACCS), pp-18-22, volume-1,IEEE, 2010.

[12] Jaisankar N and R.Saravanan, "An Extended AODV Protocol for Multipath Routing in MANETs", IACSIT International Journal of Engineering and Technology, Volume- 2, No.4, August 2010.

[13] Kaur Deep ,Kirandeep kaur, Vishal Arora, "QoS in WLAN using IEEE802.11e", 2012 Second International Conference on Advanced Computing \& Communication Technologies, IEEE Computer Society, 2012.

[14] Korkmaz Turgay, "Verifying Physical Presence of Neighbors against Replay-based Attacks in Wireless Ad Hoc Networks", Information Technology: Coding and Computing, International Journal of Information Technology, Volume- 2, No. 2, pp 704-709, April 2005.

[15] Kumar Raj, K. Duraiswamy," A Fault Tolerant Congestion Aware Routing Protocol for Mobile Adhoc Networks", Journal of Computer Science volume- 8, issue- 5, pp 673-680, 2012.

[16] Kuppusam P, Dr.K.Thirunavukkarasu, Dr.B.Kalaavathi, "A Study and Comparison of OLSR, AODV and TORA Routing Protocols in Ad Hoc Networks", pp-143 - 147, volume-5, ICECT, IEEE, 2011.

[17] Lee Sung-Hee, Young-Bae Ko, Youg-Geun Hong, and Hyoung-Jun Kim, "A New MIMC Routing Protocol Compatible with IEEE 802.11s based WLAN Mesh Networks'”, pp-126-131, ICOIN, IEEE, 2011.

[18] Llewellyn Larry C., Kenneth M. Hopkinson, Member, IEEE, and Scott R. Graham, Member, IEEE, "Distributed Fault-Tolerant Quality of Wireless Networks", IEEE Transactions On Mobile Computing, volume- 10, issue2, pp 175-190, FEBRUARY 2011.

[19] Mamatha G.S.," TODV: Performance Analysis of a Time on Demand Distance Vector Protocol for MANETS", International Journal of Computer Applications, Volume28, Issue- 2, Article 6, pp 32-36, August 2011. 